\title{
GRAPH EFFECTIVE RESISTANCE AND DistRIBUTED CONTROL: SPECTRAL PRoperties AND APPliCATIONS
}

\author{
Prabir Barooah João P. Hespanha
}

\begin{abstract}
We introduce the concept of matrix-valued effective resistance for undirected matrix-weighted graphs. Effective resistances are defined to be the square blocks that appear in the diagonal of the inverse of the matrix-weighted Dirichlet graph Laplacian matrix. However, they can also be obtained from a "generalized" electrical network that is constructed from the graph, and for which currents, voltages and resistances take matrix values.

Effective resistances play an important role in several problems related to distributed control and estimation. They appear in least-squares estimation problems in which one attempts to reconstruct global information from relative noisy measurements; as well as in motion control problems in which agents attempt to control their positions towards a desired formation, based on noisy local measurements. We show that in either of these problems, the effective resistances have a direct physical interpretation.

We also show that effective resistances provide bounds on the spectrum of the graph Laplacian matrix and the Dirichlet graph Laplacian. These bounds can be used to characterize the stability and convergence rate of several distributed algorithms that appeared in the literature.
\end{abstract}

\section{INTRODUCTION}

This paper considers undirected graphs with a weight associated with each one of its edges. The edge-weights are symmetric positive definite matrices. For such graphs we introduce the concept of "effective resistances." The effective resistance of a node is defined to be a square matrix block that appears in the diagonal of the inverse of the matrix-weighted Dirichlet graph Laplacian matrix (cf. Section II). The terminology "effective resistance" is motivated by the fact that these matrices also define a linear map from currents to voltages in a generalized electrical network that can be constructed from the undirected matrix-weighted graph. However, the voltages, currents, and resistances in this generalized electrical network take matrix values [1].

Effective resistances in "regular" electrical networks, where currents voltages and resistances are scalar valued, have been known to have far reaching implications in a variety of problems. Recurrence and transience in random walks in infinite networks [2] and the coverage and commute times of random walks in graphs [3] can be determined by the effective resistance. There is a also a strong connection between effective resistances and estimation error variances that arise in the estimation of scalar-valued variables using

This material is based upon work supported by the Institute for Collaborative Biotechnologies through grant DAAD19-03-D-0004 from the U.S. Army Research Office and by the National Science Foundation under Grant No. CCR-0311084.

Both authors are with the Dept. of Electrical and Computer Engineering and the Center for Control, Dynamical-Systems, and Computation at Univ. of California, Santa Barbara, CA 93106. relative measurements [4]. It was later shown by Barooah and Hespanha [5] that this analogy can be extended to vector measurements with matrix-valued covariances, by introducing generalized electrical networks with matrixvalued currents, voltages, and resistances. Correspondingly, the effective resistance in a generalized electrical network is matrix-valued. Every graph with positive definite matrix weights can be thought of as a generalized electrical network where the edge weights are the inverse-resistances.

The effective resistances of matrix-weighted graphs play an important role in several problems related to distributed control and estimation. We justify this statement in Section IV by presenting several such problems. The first of these problems is formulated in Section IV-A and consists of estimating a certain number of variables, based on noisy "relative" measurements. The adjective "relative" refers to the fact that the measurements only provide information about pairwise differences between the unknown variables. This problem was considered in [4-7] and has multiple applications to sensor networks, including time synchronization and sensor localization. It turns out that the covariance matrices of the estimation errors are given by the effective resistances of a graph that describes the measurement model.

A second problem is formulated in Section IV-B and consists of controlling a group of mobile agents towards a formation defined by the desired relative positions between the agents. Each agent has available for control noisy measurements of its relative position with respect to a small set of neighbors. We consider a proportional negativefeedback control law that drives each agent to the current best estimate of where it should be, based on the current positions of its neighbors. For this law, the steady-state covariance matrices of the vehicles positions are given by the effective resistances of a graph that describes the available measurements.

In Section IV-C we consider the control of a swarm of vehicles based on relative measurements. We consider the setup introduced by Fax and Murray [8] and show that stability of the group of vehicles is guaranteed when the Nyquist plot of the open-loop system does not encircle any point in a line segment between the symmetric of the sum of the traces of the effective resistances and the origin of the complex plane. A similar condition could be developed for the formation control problem in Yadlapalli et al. [9]. The stability condition in terms of effective resistances is typically more conservative than the one that appeared in [8], but it is useful because effective resistances 
have a physical interpretation that can be used to build intuition. More importantly, it is possible to construct upperand low-bounds on the effective resistances for large-scale graphs [1].

\section{GRAPH EFFECTIVE RESISTANCES}

An undirected matrix-weighted graph is a triple $\mathbf{G}=$ $(\mathbf{V}, \mathbf{E}, \mathbf{W})$, where $\mathbf{V}$ is a set of $n$ vertices; $\mathbf{E} \subset \mathbf{V} \times \mathbf{V}$ a set of $m$ edges; and $\mathbf{W}:=\left\{W_{u, v} \in \mathbb{R}^{k \times k}:(u, v) \in \mathbf{E}\right\}$ a set of symmetric positive definite matrix-valued weights for the edges of G. Since we are dealing with undirected graphs, the pairs $(u, v)$ and $(v, u)$ denote the same edge. For simplicity of notation, we exclude the existence of multiple edges between the same pair of nodes and also edges from a node to itself.

The matrix-weighted Laplacian of $\mathbf{G}$ is a $n k \times n k$ matrix $L$ with $k$ rows and $k$ columns per node such that the $k \times k$ block of $L$ corresponding to the $k$ rows associated with node $u \in \mathbf{V}$ and the $k$ columns associated with node $v \in \mathbf{V}$ is equal to

$$
\begin{cases}\sum_{v \in \mathcal{N}_{u}} W_{u, v} & u=v \\ -W_{u, v} & (u, v) \in \mathbf{E} \\ 0 & (u, v) \notin \mathbf{E} .\end{cases}
$$

where $\mathcal{N}_{u} \subset \mathbf{V}$ denotes the set of neighbors of $u$, i.e., the set of nodes that have an edge in common with $u$.

The matrix-weighted Dirichlet or Grounded Laplacian is obtained from the Laplacian by removal of rows and columns. In particular, given a subset $\mathbf{V}_{o} \subset \mathbf{V}$ consisting of $n_{o} \leq n$ nodes, the matrix-weighted Dirichlet Laplacian for the boundary $\mathbf{V}_{o}$ is a $\left(n-n_{o}\right) k \times\left(n-n_{o}\right) k$ matrix $L_{o}$ obtained from the matrix-weighted Laplacian of $\mathbf{G}$ by removing all rows and columns corresponding to the nodes in $\mathbf{V}_{o}$.

The usual graph Laplacian is a special case of the matrixweighted Laplacian when $k=1$ and all the weights are equal to one. The submatrix $L_{o}$ of $L$ in the special case of $k=1$ is called the Dirichlet Laplacian, since it arises in the numerical solution of PDE's with Dirichlet boundary conditions [10]. It is also called a Grounded Laplacian since it arises in the computation of node potentials in a electrical network. Our terminology is derived from this history, but as we shall see shortly, the matrix-weighted Dirichlet Laplacian also arises in several distributed control/estimation problems.

We say that a graph $\mathbf{G}$ is connected to $\mathbf{V}_{o}$ if there is a path from every node in the graph to at least one of the boundary nodes in $\mathbf{V}_{o}$. Lemma 1 at the end of this section shows that the Dirichlet Laplacian $L_{o}$ is invertible if and only if $\mathbf{G}$ is connected to $\mathbf{V}_{o}$.

We now formally define effective resistance of a node in a connected graph $\mathbf{G}$ : node $u$ 's effective resistance to $\mathbf{V}_{o}$, denoted by $R_{u}^{\mathrm{eff}}\left(\mathbf{V}_{o}\right)$, is the $k \times k$ block in the main diagonal of $L_{o}^{-1}$ corresponding to the $k$ rows/columns associated with the node $u \in \mathbf{V}$. This terminology is justified by the fact that these matrices also express a map from (matrix-valued) currents to (matrix-valued) voltages in an appropriately defined electrical network (cf. [1]). However, for now we are mostly interested in the fact that these effective resistances have a direct physical relevance in many distributed control/estimation problems. Moreover, they also allow us to deduce properties of the spectrum of the matrix-weighted Dirichlet Laplacian and even properties of the spectrum of the original matrix-weighted Laplacian. It should be noted that these effective resistances are matrixvalued.

The previous definitions relied on the non-singularity of $L_{o}$, which is established by the following lemma.

Lemma 1 (Invertibility). The matrices $L_{o}$ and $L$ are both positive semi-definite. Moreover, the matrix $L_{o}$ is positive definite if and only if $\mathbf{G}$ is connected to $\mathbf{V}_{o}$.

To proceed with the proof of this lemma as well as to build further insight into the structure of the matrices $L$ and $L_{o}$, we note that the matrix-weighted Laplacian can also be defined compactly using the incidence matrix of the graph. To do so, we consider a directed graph $\overrightarrow{\mathbf{G}}$ obtained by assigning arbitrary directions to the edges of $\mathbf{G}$. The incidence matrix of the directed graph $\overrightarrow{\mathbf{G}}$ with $n$ nodes and $m$ edges is an $n \times m$ matrix with one row per node and one column per edge defined by $A:=\left[a_{u e}\right]$, where $a_{u e}$ is nonzero if and only if the edge $e \in \mathbf{E}$ is incident on the node $u \in \mathbf{V}$. When nonzero, $a_{u e}=-1$ if the edge $e$ is directed towards $u$ and $a_{u e}=1$ otherwise. Define $\mathcal{W}=$ $\operatorname{diag}\left(W_{1}, \ldots, W_{m}\right)$. Figure 1 shows a graph $\mathbf{G}$, a possible corresponding directed graph $\overrightarrow{\mathbf{G}}$ and its associated incidence matrix. The matrix-weighted Laplacian of the graph $\mathbf{G}$ can be expressed as

$$
L:=\mathcal{A} \mathcal{W} \mathcal{A}^{T}, \quad \mathcal{A}=A \otimes I_{k}
$$

where $\otimes$ denotes the Kronecker product and $I_{k}$ is a $k \times k$ identity matrix. Note that the matrix-weighted Laplacian does not depend on the directions of the edges chosen to define the incidence matrix.

The Dirichlet Laplacian can also be expressed compactly as

$$
L_{o}:=\mathcal{A}_{o} \mathcal{W} \mathcal{A}_{o}^{T}, \quad \mathcal{A}_{o}:=A_{o} \otimes I_{k},
$$

where the generalized basis incidence matrix $A_{o}$ is a matrix obtained by removing all the rows from the incidence matrix $A$ that correspond to nodes in $\mathbf{V}_{o}$. Figure 1 also shows a graph $\mathbf{G}$ and its associated matrix-weighted Laplacian $L$ when every edge weight is equal to the identity matrix.

Proof of Lemma 1. The incidence matrix $A \in \mathbb{R}^{n \times m}$ has a row rank less than or equal to $n-1$ [11, Theorem 8.3.1], and since $\mathcal{W}$ is positive definite, $L$ is positive semi-definite. The matrix $A_{o}$ is full-row rank if and only if $\mathbf{G}$ is connected to $\mathbf{V}_{o}$ [1], which establishes the condition for $L_{o}$ to be positive definite. 


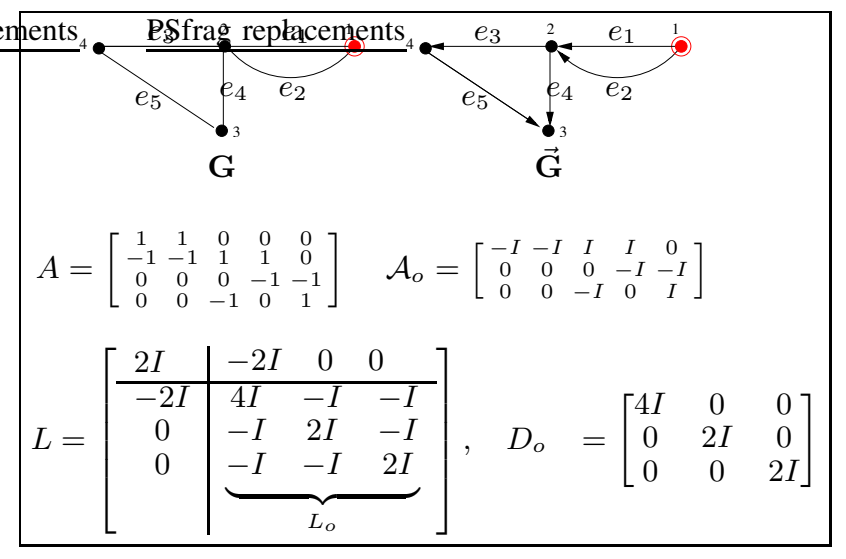

Fig. 1. A graph $\mathbf{G}$, a directed version $\overrightarrow{\mathbf{G}}$ and its incidence matrix $A$ (row and column indices of $A$ correspond to node and edge indices, respectively). The matrix $\mathcal{A}_{o}$ is for the boundary with the single node $\mathbf{V}_{o}=\{1\}$. The matrix-weighted Laplacian is shown for the case when all the edge weights are equal to the identity matrix.

\section{EFFECTIVE RESISTANCE VS. SPECTRAL PROPERTIES}

The effective resistances of a graph can be uses to study several spectral properties of a graph. The following result provides the starting point to establish these results.

Lemma 2 (Spectrum of $L$ and $L_{o}$ ). Assume that $\mathbf{G}$ is connected to $\mathbf{V}_{o}$ and denote by $\lambda_{1}(L) \leq \lambda_{2}(L) \leq \cdots \leq$ $\lambda_{n k}(L)$ the sorted eigenvalues of the $L$ and by $\lambda_{1}\left(L_{o}\right) \leq$ $\lambda_{2}\left(L_{o}\right) \leq \cdots \leq \lambda_{\left(n-n_{o}\right) k}\left(L_{o}\right)$ the sorted eigenvalues of $L_{o}$. For every $i \in\left\{1,2 \ldots,\left(n-n_{o}\right) k\right\}$

$$
\lambda_{i}\left(L_{o}\right) \geq \frac{1}{\sum_{u \in \mathbf{V}} \operatorname{trace} R_{u}^{\text {eff }}\left(\mathbf{V}_{o}\right)}
$$

and

$$
\lambda_{i}(L) \leq \lambda_{i}\left(L_{o}\right) \leq \lambda_{i+k n_{o}}(L) .
$$

Proof. The inequality (3) is a consequence of the fact that any eigenvalue of the positive definite matrix $L_{o}^{-1}$ can be upper-bounded by its trace, which can be obtained by adding up all the traces of its diagonal blocks $R_{u}^{\text {eff }}\left(\mathbf{V}_{o}\right)$, $u \in \mathbf{V}$. This means that every eigenvalue of $L_{o}^{-1}$ satisfies:

$$
\lambda_{i}\left(L_{o}^{-1}\right) \leq \operatorname{trace} L_{o}^{-1}=\sum_{u \in \mathbf{V}} \operatorname{trace} R_{u}^{\mathrm{eff}}\left(\mathbf{V}_{o}\right),
$$

from which (3) follows since the eigenvalues of $L_{o}^{-1}$ and $L_{o}$ are reciprocals of each other. The inequality (4) is a direct application of the Interlacing Eigenvalues Theorem [12, Theorem 4.3.15].

By construction, the matrix-weighted Laplacian $L$ has the property that $L\left(\left[I_{k}, \ldots, I_{k}\right]^{T}\right)_{n k \times k}=0$, where $I_{k}$ denotes the $k \times k$ identity matrix. This means that $L$ has $k$ eigenvalues equal to zero. The effective resistances can be used to obtain a lower bound on the smallest non-zero eigenvalue of $L$. In particular, when $n_{o}=1$, we conclude from (3) and (4) that

$$
\lambda_{1+k}(L) \geq \lambda_{1}\left(L_{o}\right) \geq \frac{1}{\sum_{u \in \mathbf{V}} \operatorname{trace} R_{u}^{\text {eff }}\left(\mathbf{V}_{o}\right)},
$$

which provides a lower-bound on the smallest strictly positive eigenvalue of $L$. The following lemma summarizes the discussion above:

Lemma 3 (Albegraic Connectivity). For any single-node boundary set $\mathbf{V}_{o}$, for which $\mathbf{G}$ is connected, the matrix $L$ has exactly $k$ zero eigenvalues and all remaining eigenvalues satisfy

$$
\lambda_{i}(L) \geq \frac{1}{\sum_{u \in \mathbf{V}} \operatorname{trace} R_{u}^{\mathrm{eff}}\left(\mathbf{V}_{o}\right)}>0 .
$$

When $k=1$ and all the edge-weights are equal to one, the matrix $L$ is the usual graph Laplacian and the smallest nonzero eigenvalue is known as the algebraic connectivity of the graph. The algebraic connectivity is a measure of performance/speed of consensus algorithms [13].

The convergence of several discrete-time distributed algorithms is determined by the spectrum of the matrix

$$
J_{o}:=I-\gamma D_{o}^{-1} L_{o}, \quad \gamma \in(0,1],
$$

where $D_{o}$ is a block diagonal matrix that contains the $k \times k$ blocks that appear in the diagonal of the matrix-weighted Dirichlet Laplacian $L_{o}$ (see Fig. 1 for an example). The next lemma establishes the stability of $J_{o}$ and provides a bound on the spectrum of this matrix.

Lemma 4 (Discrete-time convergence). Assume that $\mathbf{G}$ is connected to $\mathbf{V}_{o}$. Every eigenvalue of $J_{o}$ is real and satisfies

$$
-1<\lambda_{i}\left(J_{o}\right) \leq 1-\frac{\gamma}{\lambda_{\max }\left(D_{o}\right) \sum_{u \in \mathbf{V}} \operatorname{trace} R_{u}^{\mathrm{eff}}\left(\mathbf{V}_{o}\right)},
$$

where $\lambda_{\max }\left(D_{o}\right)$ denotes the largest eigenvalue of $D_{o}$. Moreover, when the weighting matrices $W_{u, v},(u, v) \in \mathbf{E}$ are all diagonal or all equal (but not necessarily diagonal), we actually have

$$
\left|\lambda_{i}\left(J_{o}\right)\right| \leq 1-\frac{\gamma}{\lambda_{\max }\left(D_{o}\right) \sum_{u \in \mathbf{V}} \operatorname{trace} R_{u}^{\text {eff }}\left(\mathbf{V}_{o}\right)} .
$$

Proof. Since

$$
J_{o}=D_{o}^{-\frac{1}{2}}\left(I-\gamma D_{o}^{-\frac{1}{2}} L_{o} D_{o}^{-\frac{1}{2}}\right) D_{o}^{\frac{1}{2}},
$$

we conclude that $J_{o}$ can be transformed by a similarity transformation into the symmetric matrix $I-\gamma D_{o}^{-\frac{1}{2}} L_{o} D_{o}^{-\frac{1}{2}}$ and therefore all eigenvalues of $J_{o}$ must be real. Moreover, all eigenvalues of $J_{o}$ have absolute value strictly smaller than one because the discrete-time Lyapunov equation

$$
J_{o}^{\prime} P J_{o}-P=-Q
$$

has a positive definite solution $P:=D_{o} L_{o}^{-1} D_{o}>$ for

$$
Q:=\gamma\left(2 D_{o}-L_{o}\right)+\gamma(1-\gamma) L_{o} \geq \gamma\left(2 D_{o}-L_{o}\right)>0 .
$$

To verify that this matrix is positive definite, we note that $2 D_{o}-L_{o}$ is very similar to the matrix-weighted Laplacian $L$, and in fact, $2 D_{o}-L_{o}=\left|\mathcal{A}_{o}\right| \mathcal{W}\left|\mathcal{A}_{o}^{T}\right|$, where $\left|\mathcal{A}_{o}\right|$ is a matrix whose entries are the absolute values of the entries of $\mathcal{A}_{o}$. The positive definiteness of $\left|\mathcal{A}_{o}\right| \mathcal{W}\left|\mathcal{A}_{o}^{T}\right|$ can be proved in a manner similar to that used in proving the positive definiteness of $L_{o}=\mathcal{A}_{o} \mathcal{W} \mathcal{A}_{o}^{T}$ (Lemma 1). 
Denoting by $\lambda \in(-1,1)$ an eigenvalue of $J_{o}$ and by $x$ the corresponding eigenvector, we have that

$$
\left(I-\gamma D_{o}^{-1} L_{o}\right) x=\lambda x \quad \Leftrightarrow \quad L_{o}^{-1} D_{o} x=\frac{\gamma}{1-\lambda} x .
$$

Therefore

$$
\frac{\lambda_{\max }\left(D_{0}\right)}{\lambda_{\min }\left(L_{o}\right)}\|x\| \geq\left\|L_{o}^{-1} D_{o} x\right\|=\frac{\gamma}{1-\lambda}\|x\|,
$$

from which we conclude using Lemma 2 that

$$
\lambda \leq 1-\gamma \frac{\lambda_{\min }\left(L_{o}\right)}{\lambda_{\max }\left(D_{0}\right)} \leq 1-\frac{\gamma}{\lambda_{\max }\left(D_{0}\right) \sum_{u \in \mathbf{V}} \operatorname{trace} R_{u}^{\mathrm{eff}}\left(\mathbf{V}_{o}\right)},
$$

which proves (5).

When all the weighting matrices are diagonal, the matrix $J_{o}$ is non-negative for every $\gamma \leq 1$. Therefore by Perron's Theorem [12, Theorem 8.3.1] we know that its eigenvalue with the largest absolute value must be a positive number. The last inequality in the Lemma then follows from (5). When all weighting matrices are equal to some $W>0$, it follows from the definition (2) that

$$
J_{o}=\bar{J}_{o} \otimes I_{k},
$$

where $J_{o}:=I-\gamma\left(\bar{D}_{o}^{-1} \bar{L}_{o}\right) \in \mathbb{R}^{\left(n-n_{o}\right) \times\left(n-n_{o}\right)}, \bar{L}_{o}$ is the Dirichlet Laplacian for scalar weights equal to 1 , and $\bar{D}_{o}$ its main diagonal. Thus, the weights $W$ play no role. The matrix $\bar{D}_{o}^{-1} \bar{L}_{o}$ is nonnegative, and we conclude from the reasoning above that its eigenvalue with largest absolute value is positive. Since $J_{o}=\bar{J}_{o} \otimes I_{k}$, the distinct eigenvalues of $J_{o}$ are just the distinct eigenvalues of $\bar{J}_{o}$, so we conclude that the eigenvalue of $J_{o}$ with largest norm is also positive. The last inequality again follows from (5).

The convergence of several continuous-time distributed algorithms is determined by the spectrum of the matrix

$$
G_{o}:=-\gamma D_{o}^{-1} L_{o}, \quad \gamma>0
$$

where $D_{o}$ is a block diagonal matrix that contains the $k \times$ $k$ blocks on the diagonal of the matrix-weighted Dirichlet Laplacian $L_{o}$.

Lemma 5 (Continuous-time convergence). Assume that $\mathbf{G}$ is connected to $\mathbf{V}_{o}$. Every eigenvalue of $G_{o}$ is real and satisfies

$$
\lambda_{i}\left(G_{o}\right) \leq-\frac{\gamma}{\lambda_{\max }\left(D_{o}\right) \sum_{u \in \mathbf{V}} \operatorname{trace} R_{u}^{\mathrm{eff}}\left(\mathbf{V}_{o}\right)},
$$

where $\lambda_{\max }\left(D_{o}\right)$ denotes the largest eigenvalue of $D_{o}$.

Proof. Since

$$
G_{o}=D_{o}^{-\frac{1}{2}}\left(-\gamma D_{o}^{-\frac{1}{2}} L_{o} D_{o}^{-\frac{1}{2}}\right) D_{o}^{\frac{1}{2}},
$$

we conclude that $G_{o}$ can be transformed by a similarity transformation into the negative definite matrix $-\gamma D_{o}^{-\frac{1}{2}} L_{o} D_{o}^{-\frac{1}{2}}$ and therefore all eigenvalues of $G_{O}$ must be real and negative. Moreover, defining $P:=\frac{\gamma}{2} L_{o}^{-1}$ we conclude that

$$
\left(\alpha I+G_{o}\right) P+P\left(\alpha I+G_{o}\right)^{\prime}=\gamma\left(\alpha L_{o}^{-1}-\gamma D_{o}^{-1}\right) .
$$

The matrix on the right hand side is then negative definite as long as

$$
\gamma \lambda_{\min }\left(D_{o}^{-1}\right)>\alpha \lambda_{\max }\left(L_{o}^{-1}\right) \Leftrightarrow \alpha<\gamma \frac{\lambda_{\min }\left(L_{o}\right)}{\lambda_{\max }\left(D_{o}\right)} .
$$

From Lyapunov's Theorem, we thus conclude that $\alpha I+G_{o}$ is Hurwitz for every $\alpha<\frac{\gamma \lambda_{\min }\left(L_{o}\right)}{\lambda_{\max }\left(D_{o}\right)}$, which implies that the eigenvalue of $G_{o}$ must be more negative than $\frac{\gamma \lambda_{\min }\left(L_{o}\right)}{\lambda_{\max }\left(D_{o}\right)}$. The inequality (6) follows from this and Lemma 2.

\section{APPliCATIONS TO DISTRIBUTED CONTROL AND ESTIMATION}

We now describe a few problems in distributed control and estimation for which the matrix-weighted Dirichlet Laplacian and the effective resistances play a key role.

\section{A. Graph estimation}

Consider the problem of estimating the values of $n$ node variables $x_{1}, x_{2}, \ldots x_{n} \in \mathbb{R}^{k}, k \geq 1$ from noisy "relative" measurements of the form

$$
y_{u, v}=x_{u}-x_{v}+\epsilon_{u, v}, \quad(u, v) \in \mathbf{E} \subset \mathbf{V} \times \mathbf{V},
$$

where the $\epsilon_{u, v}$ 's are uncorrelated zero-mean noise vectors with associated covariance matrices $P_{u, v}=\mathrm{E}\left[\epsilon_{u, v} \epsilon_{u, v}^{\prime}\right]$. This estimation problem is relevant for such wide ranging applications such as location estimation and time synchronization in sensor networks $[4,6,7]$

This estimation problem can be associated with a matrixweighted graph $\mathbf{G}=(\mathbf{V}, \mathbf{E}, \mathcal{W})$ with $n$ nodes and $m$ edges, with node set $\mathbf{V}:=\{1,2, \ldots, n\}$, edge set $\mathbf{E}$ consisting of all the pairs of nodes $(u, v)$ for which a noisy measurement of the form (7) is available; and weight set $\mathcal{W}$ consisting of the inverses of the covariance matrices $W_{u, v}:=P_{u, v}^{-1}$, $(u, v) \in \mathbf{E}$.

Just with relative measurements, determining the $x_{u}$ 's is only possible up to an additive constant. To avoid this ambiguity, we assume that at least one of the nodes is used as a reference and therefore its node variable can be assumed known. In general, several node variables may be known and therefore we may have several references. We denote by $\mathbf{V}_{o}$ the set of reference nodes. This problem was introduced in [5] for a single reference node.

By stacking together all the measurements into a single vector $y \in \mathbb{R}^{k m}$, all node variables (known and unknown) into one vector $X \in \mathbb{R}^{k n}$, and all the measurement errors into a vector $\epsilon \in \mathbb{R}^{k m}$, we can express all the measurement equations (7) in the following compact form:

$$
y=\mathcal{A}^{T} X+\epsilon,
$$

where $\mathcal{A}$ is as defined in (1). By partitioning $X$ into a vector $x$ containing all the unknown node variables and another vector $r$ containing all the known reference node variables, we can re-write (8) as

$$
y=\mathcal{A}_{r}^{T} r+\mathcal{A}_{o}^{T} x+\epsilon,
$$

where $A_{r}$ contains the rows of $A$ corresponding to the reference nodes and $A_{o}$ contains the rows of $A$ corresponding to the unknown node variables (see Figure 1 for an example). 
The estimation of the unknown $x$ based on the linear measurement model (9) is a classical estimation problem. The Best Linear Unbiased Estimator (BLUE) of $x$ is given by

$$
\hat{x}:=L_{o}^{-1} b, L_{o}:=\mathcal{A}_{o} \mathcal{W} \mathcal{A}_{o}^{T}, b:=\mathcal{A}_{o} \mathcal{W}\left(y-\mathcal{A}_{r}^{T} r\right),
$$

where $\mathcal{A}_{o}$ is as defined in (2) and $\mathcal{W} \in \mathbb{R}^{k m \times k m}$ is a blockdiagonal matrix with inverse-covariances in the diagonal $\mathcal{W}=\operatorname{diag}\left(P_{1}^{-1}, \ldots, P_{m}^{-1}\right)$;

Among all linear estimators of $x,(10)$ has the smallest variance for the estimation error $x-\hat{x}$ and the inverse of $L_{o}$ provides the covariance matrix of the estimation error [14]:

$$
\mathrm{E}\left[(x-\hat{x})(x-\hat{x})^{\prime}\right]=L_{o}^{-1}
$$

As we saw before, the inverse of $L_{o}$ exists as long as the graph $\mathbf{G}$ is connected to $\mathbf{V}_{o}$.

From (11), we also conclude that the covariance matrix $\Sigma_{u}$ of the estimation error for the variable $x_{u}$ appears in the corresponding $k \times k$ diagonal block of $L_{o}^{-1}$, which is precisely node $u$ 's effective resistance to $\mathbf{V}_{o}$ defined in Section II. For large graphs one is interested in how the covariance of the BLU estimate grows as a function of distance from the reference node. This question is answered in [1] by determining how the matrix-effective resistance scales with distance for a large class of graphs.

A distributed algorithms for the computation of the estimates have been proposed in [7]. A similar algorithm is also proposed in [6]. This algorithm is based on the observation that the optimal estimate $\hat{x}$ in (10) is a solution to the following equation

$$
\hat{x}=J_{o} \hat{x}+\gamma D_{o}^{-1} \hat{b}
$$

where $J_{o}:=I-\gamma D_{o}^{-1} L_{o}, \gamma \in(0,1]$. Since all eigenvalues of $J_{o}$ have absolute value smaller than one (cf. Lemma 4), the discrete-time system

$$
\hat{x}(t+1)=J_{o} \hat{x}(t)+\gamma D_{o}^{-1} \hat{b}
$$

will converge precisely to the solution to (12), which is the optimal estimate $\hat{x}$. It turns out that the recursion in (12) can be computed in a distributed fashion. In practice, each node only computes the $k$ elements of $\hat{x}(t)$ that correspond to its node variable. Since the $k$ rows of $J_{o}$ that corresponds to the node $u$ only have nonzero $k \times k$ off-diagonal blocks in the positions corresponding to the neighbors of $u$, all that $u$ needs are the current estimates of its 1-hop neighbors. The algorithm proposed in [7] is actually a special case of this one for $\gamma=1$. Lemma 4 relates the speed of convergence of this algorithm with the effective resistances.

\section{B. Formation control with noisy measurements}

Consider a group of $n$ mobile agents moving in $k$ dimensional space that one desires to control to a given formation defined by their relative positions. In particular, denoting by $x_{u} \in \mathbb{R}^{k}, u \in \mathbf{V}:=\{1,2, \ldots, n\}$ the position of the $u$ th agent, the control objective is to make the positions converge to values for which

$$
x_{u}-x_{v}=r_{u, v}, \quad \forall(u, v) \in \mathbf{V} \times \mathbf{V},
$$

where $r_{u, v}$ denotes the desired relative position of agent $u$ with respect to agent $v$. One of the agents $o \in \mathbf{V}$ will be called the leader and it will move independently of the remaining ones. The remaining agents attempt to maintain the formation specified by (13). The leader may actually not be a physical agent. Instead, it may be a "reference" that is known to at least one of the physical agents.

Not all agents are able to measure their relative positions with respect to all other agents and therefore each agent is constrained to use only a few relative position measurements to compute its control signal. We denote by $\mathbf{E} \subset \mathbf{V} \times \mathbf{V}$ the set of pairs of agents that can measure their relative positions. In particular, the existence of a pair $(u, v)$ in $\mathbf{E}$ signifies that agent $u$ can measure its position with respect to $v$ and similarly, $v$ can measure its position with respect to $u$, although both measurements will be corrupted with noise. Since the noise corrupting the measurement of $x_{u}-x_{v}$ available to $u$ will be in general different from the noise on the measurement of $x_{v}-x_{u}$ available to $v$, we need to distinguish these two measurements. To this end, we introduce a directed edge set $\overrightarrow{\mathbf{E}}$ containing the two ordered pairs $(u, v),(v, u)$ whenever $(u, v) \in \mathbf{E}$. We assume that a noisy measurement $y_{u, v}$ of the following form is available to agent $u$ if $(u, v) \in \overrightarrow{\mathbf{E}}$ :

$$
y_{u, v}=x_{u}-x_{v}+\epsilon_{u, v}
$$

where $\epsilon_{u, v}$ is a white random noise process with autocorrelation matrix given by $\mathrm{E}\left[\epsilon_{u, v}\left(t_{1}\right) \epsilon_{u, v}^{T}\left(t_{2}\right)\right]=\delta\left(t_{1}-t_{2}\right) R_{u, v}$.

Note that by assumption, if a measurement $y_{u, v}$ is available to $u$, then the measurement $y_{v, u}$ is available to $v$. The noise processes over different edges are assumed independent of each other. In particular, $e_{u, v}(t)$ is independent of $e_{v, u}(t)$ for all $t$. In case $x_{o}$ is a reference and not a physical agent, an edge between the node $u$ and the leader $o$ means that the physical agent $u$ is able to measure its position with respect to the reference $o$.

The problem above is now associated with a matrixweighted directed graph $\overrightarrow{\mathbf{G}}=(\mathbf{V}, \overrightarrow{\mathbf{E}}, \overrightarrow{\mathbf{W}})$ with node set $\mathbf{V}=\{1,2, \ldots, n\}$; directed edge set $\overrightarrow{\mathbf{E}}$ consisting of all ordered pairs of nodes $(u, v)$ for which a noisy measurement of the form (14) is available; and weight set $\overrightarrow{\mathbf{W}}$ consisting of the inverses of the autocorrelation matrices $W_{u, v}:=R_{u, v}^{-1}$, $(u, v) \in \overrightarrow{\mathbf{E}}$. We assume that even though the measurement errors on the two edges $(u, v)$ and $(v, u)$ connecting the nodes $u$ and $v$ are uncorrelated, they have the same autocorrelation matrix; i.e., $R_{u, v}=R_{v, u}$. We will refer to this assumption, together with the assumption that the directed edge $(u, v)$ exists iff $(v, u)$ exists, as bidirectionality. Fig. 2 shows an example of a bidirectional directed graph and its associated undirected graph.

We are interested in control laws for which each agent uses all its measurements to construct an optimal estimate of the difference between its currently position and what this "should" be, in view of what it know about its neighbors positions. The measurements available to an arbitrary agent $u \in \mathbf{V}$ are

$$
y_{u, v}=x_{u}-x_{v}+\epsilon_{u, v}, \quad \forall v \in \mathcal{N}_{u},
$$


where $\mathcal{N}_{u} \subset \mathbf{V}$ denotes set of nodes $v$ such that $(u, v) \in$ $\overrightarrow{\mathbf{E}}$. If agent $u$ assumes that all its neighbors are correctly positioned then, according to (13), the desired position of $u$ is given by any one of the following equations

$$
x_{u}^{d}=x_{v}+r_{u, v}, \quad \forall v \in \mathcal{N}_{u} .
$$

Combining the two previous sets of equations, we obtain

$$
y_{u, v}=x_{u}-x_{u}^{d}+r_{u, v}+\epsilon_{u, v}, \quad \forall v \in \mathcal{N}_{u},
$$

from which agents $u$ estimates its position error $x_{u}-x_{u}^{d}$. It is straightforward to show that the Best Linear Unbiased estimate of $x_{u}-x_{u}^{d}$ is given by $D_{u}^{-1} \sum_{v \in \mathcal{N}_{u}} R_{u, v}^{-1}\left(y_{u, v}-\right.$ $\left.r_{u, v}\right)$, where $D_{u}:=\sum_{v \in \mathcal{N}_{u}} R_{u, v}^{-1}$. This motivates the following negative proportional control law for the agents

$$
\dot{x}_{u}=-\gamma D_{u}^{-1} \sum_{v \in \mathcal{N}_{u}} R_{u, v}^{-1}\left(y_{u, v}-r_{u, v}\right), \quad \forall u \in \mathbf{V} \backslash\{o\},
$$

where $\gamma$ denotes some positive number. For analysis purposes it is convenient to describe the system dynamics in term of positions with respect to the leader. Defining $\tilde{x}_{u}=x_{u}-x_{o}$, one concludes that

$$
\dot{\tilde{x}}_{u}=-\gamma D_{u}^{-1} \sum_{v \in \mathcal{N}_{u}} R_{u, v}^{-1}\left(\tilde{x}_{u}-\tilde{x}_{v}-r_{u, v}+\epsilon_{u, v}\right)-\dot{x}_{o},
$$

for every $u \in \mathbf{V} \backslash\{o\}$. By stacking all the positions $\tilde{x}_{u}$, $u \in \mathbf{V} \backslash\{o\}$ in a column vector $\tilde{x}$, the above systems can be written as follows:

$$
\dot{\tilde{x}}=-\gamma D_{o}^{-1} L_{o} \tilde{x}+\gamma D_{o}^{-1} B_{o} W(r-\epsilon)-\dot{x}_{o} \mathbf{1},
$$

where $r$ is a column vector obtained by stacking all the $r_{u, v}$ on top of each other; $\epsilon$ is a column vector obtained by stacking all the $\epsilon_{u, v} ; \mathbf{1}$ is a $n-1 \times 1$ column vector of all 1 's; $W>0$ is a block-diagonal matrix with $k$ rows/columns for each edge in $\overrightarrow{\mathbf{E}}$, with the weights $W_{u, v}:=R_{u, v}^{-1}$, $(u, v) \in \overrightarrow{\mathbf{E}}$ in the diagonal; $D_{o}>0$ is a block-diagonal matrix with $k$ rows/columns for each node in $\mathbf{V} \backslash \mathbf{V}_{o}$, with $D_{u}, u \in \mathbf{V} \backslash \mathbf{V}_{o}$ as defined earlier in the diagonal; $L_{o}=\frac{1}{2} \mathcal{A}_{o} W \mathcal{A}_{o}^{T}$ where $\mathcal{A}_{o}$ is the generalized incidence matrix for the directed graph $(\mathbf{V}, \overrightarrow{\mathbf{E}})$ with $V_{o}=\{o\}$ (cf. section II); and $B_{o}$ is a matrix with $k$ rows for each vertex in $\mathbf{V} \backslash \mathbf{V}_{o}$ and $k$ columns for each edge in $\overrightarrow{\mathbf{E}}$, constructed as follows: the $k$ columns corresponding to edge $(u, v) \in \overrightarrow{\mathbf{E}}$ are all equal to zero except for the block corresponding to the node $u$, which is equal to $I_{k}$. The white noise process $\epsilon$ has block diagonal autocorrelation matrix given by $\mathrm{E}\left[\epsilon\left(t_{1}\right) \epsilon^{T}\left(t_{2}\right)\right]=\delta\left(t_{1}-t_{2}\right) W^{-1}$. Fig. 2 shows an example of the matrices defined above.

$L_{o}$ is exactly the matrix-weighted Dirichlet Laplacian for the matrix-weighted undirected graph $(\mathbf{V}, \mathbf{E}, \mathbf{W})$ with boundary $\mathbf{V}_{o}:=\{o\}$ and with weight $W_{u, v}$ on every undirected edge $(u, v)$ assigned as the weight on the corresponding directed edge $(u, v) \in \overrightarrow{\mathbf{E}}$. Note that we get the undirected Laplacian in the system dynamic equations (15) due to the bidirectionality assumption.

From Lemma 5, we conclude that (15) is an asymptotically stable system and Lemma 5 actually relates the speed of its slowest pole with the effective resistances. It turns out that the effective resistances play an even more interesting

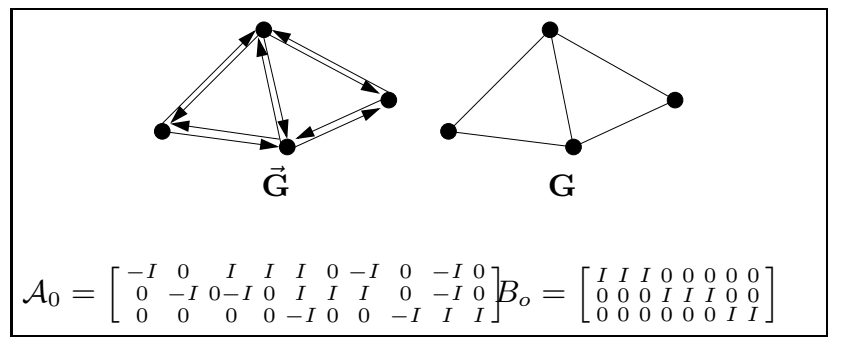

Fig. 2. A (bidirectional) directed graph $\overrightarrow{\mathbf{G}}$ and the associated undirected graph $\mathbf{G}$. The matrices $\mathcal{A}_{o}$ and $B_{o}$ shown are for the graph $\overrightarrow{\mathbf{G}}$.

role for this system. To see this, we further re-write the model (15) as

$$
\dot{\tilde{x}}=-\gamma D_{o}^{-1} L_{o} \tilde{x}+w+b,
$$

where $b:=\gamma D_{o}^{-1} B_{o} W r-\dot{x}_{o} \mathbf{1}$ and $w:=-\gamma D_{o}^{-1} B_{o} W \epsilon$ is a white noise random process with autocorrelation matrix given by

$$
\begin{aligned}
& \mathrm{E}\left[w\left(t_{1}\right) w^{T}\left(t_{2}\right)\right]=\gamma^{2} D_{o}^{-1} B_{o} W \mathrm{E}\left[\epsilon\left(t_{1}\right) \epsilon^{T}\left(t_{2}\right)\right] W B_{o}^{T} D_{o}^{-1} \\
= & \gamma^{2} \delta\left(t_{1}-t_{2}\right) D_{o}^{-1} B_{o} W B_{o}^{T} D_{o}^{-1}=\gamma^{2} \delta\left(t_{1}-t_{2}\right) D_{o}^{-1},
\end{aligned}
$$

where we used the fact that $B_{o} W B_{o}^{T}=D_{o}$. Since the Lyapunov equation

$$
-\gamma D_{o}^{-1} L_{o} \Sigma_{\infty}-\gamma \Sigma_{\infty} L_{o} D_{o}^{-1}+\gamma^{2} D_{o}^{-1}=0
$$

has a positive definite solution

$$
\Sigma_{\infty}=\frac{\gamma}{2} L_{o}^{-1}
$$

it is straightforward to show that the covariance matrix of $\tilde{x}$ converges to $\Sigma_{\infty}$. In particular, the steady-state covariance matrix of the relative position $\tilde{x}_{u}:=x_{u}-x_{o}$ is given by $k \times k$ diagonal block of $\Sigma_{\infty}$, which is given by $\gamma / 2$ times node $u$ 's effective resistance $R_{u, o}^{\text {eff }}$ to $\mathbf{V}_{o}:=\{o\}$ defined in Section II. The scaling of matrix-valued effective resistance $R_{u, o}^{\mathrm{eff}}$ as a function of distance $d_{u, o}$ of $u$ from the leader $o$ (addressed in [1]) determine how the structure of the graph $\mathbf{G}$ affect the growth of the effective resistance, and therefore tracking error covariance.

\section{Stability of multi-vehicle swarms}

Consider a group of $n$ single-input/single-output vehicles all with the same transfer function $P(s)$ from their control inputs $u_{i}, i \in \mathbf{V}:=\{1,2, \ldots, n\}$ to their positions $x_{i}$. Each vehicle is able to determine its relative position with respect to a few other vehicles. We denote by $\mathbf{E} \subset \mathbf{V} \times \mathbf{V}$ the set of pairs of vehicles that can measure each other relative positions. In particular, the existence of a pair $(i, j)$ in $\mathbf{E}$ signifies that the measurement

$$
y_{i, j}:=x_{i}-x_{j}, \quad \forall(i, j) \in \mathbf{E}
$$

is available to both vehicles $i$ and $j$. The control objective is for all vehicles to rendezvous at a point. To this effect each vehicle computes a weighted average

$$
e_{i}:=\sum_{j \in \mathcal{N}_{i}} w_{i, j}\left(x_{i}-x_{j}\right), \quad i \in \mathbf{V}
$$


of the relative positions that it can measure and uses it for feedback according to $U_{i}(s)=K(s) E_{i}(s)$, where $U_{i}(s)$ and $E_{i}(s)$ denote the Laplace transforms of $u_{i}$ and $e_{i}$, respectively; and $K(s)$ is a suitably selected controller transfer function used by every vehicle. The error equation can be associated with an undirected scalar-weighted graph $\mathbf{G}=(\mathbf{V}, \mathbf{E}, \mathbf{W})$, with weights $\mathbf{W}:=\left\{w_{i, j} \in \mathbb{R}:(i, j) \in\right.$ $\mathbf{E}\}$. It was shown by Fax and Murray [8] that the resulting closed-loop system is stable if and only if for every nonzero eigenvalue $\lambda_{i}(L)$ of the scalar-weighted Laplacian matrix $L$ of $\mathbf{G}$ there is no net encirclements of $-1 / \lambda_{i}(L)$ by the Nyquist plot of $-K(s) P(s)$. We conclude from (3) that $-1 / \lambda_{i}(L) \geq-\sum_{u \in \mathbf{V}}$ trace $R_{u}^{\text {eff }}\left(\mathbf{V}_{o}\right)$. Moreover, it can be easily seen that $2 D-L=|\mathcal{A}| \mathcal{W}\left|\mathcal{A}^{T}\right| \geq 0$ (see the proof of the discrete time convergence Lemma 4), so $\lambda_{i}(L)<2 \lambda_{\max }(D)$. We conclude that for all nonzero $\lambda_{i}(L),-1 / \lambda_{i}(L)$ lies in the range

$$
\left[\frac{-1}{\lambda_{2}(L)}, \frac{-1}{\lambda_{\max }(L)}\right] \subset\left[-\sum_{u \in \mathbf{V}} \operatorname{trace} R_{u}^{\mathrm{eff}}\left(\mathbf{V}_{o}\right),-\frac{1}{2 \lambda_{\max }(D)}\right] .
$$

We therefore conclude that the closed-loop system is stable, as long as the the Nyquist plot of $-K(s) P(s)$ does not encircle any point in the line segment from $-\sum_{u \in \mathbf{V}}$ trace $R_{u}^{\text {eff }}\left(\mathbf{V}_{o}\right)$ to $-1 / 2 \lambda_{\max }(D)$. Large effective resistances will generally lead to longer line segments and therefore more stringent stability conditions.

Similar conclusions can be drawn for the type of formations considered in [9] and [15], where every vehicle tries to maintain a constant separation between itself and its neighbors, and there is a leader whose control action does not depend on the other vehicles. In this case the quantities of interest are the eigenvalues of the Dirichlet Laplacian, $\lambda_{i}\left(L_{o}\right)$. In particular, the formation will be stable if and only if for every eigenvalue $\lambda_{i}\left(L_{o}\right)$ of the scalarweighted Dirichlet Laplacian matrix $L_{o}$ of $\mathbf{G}$ there is no net encirclements of $-1 / \lambda_{i}\left(L_{o}\right)$ by the Nyquist plot of $-K(s) P(s)$. We can derive in a manner similar to that above that all $-1 / \lambda_{i}\left(L_{o}\right)$ lies in the range

$\left[\frac{-1}{\lambda_{\min }\left(L_{o}\right)}, \frac{-1}{\lambda_{\max }\left(L_{o}\right)}\right] \subset\left[-\sum_{u \in \mathbf{V}} \operatorname{trace} R_{u}^{\mathrm{eff}}\left(\mathbf{V}_{o}\right), \frac{-1}{2 \lambda_{\max }\left(D_{o}\right)}\right]$.

\section{CONClusion}

We introduced the concept of matrix-valued effective resistance for undirected matrix-weighted graphs and showed that effective resistances have a direct physical interpretation in several problems related to distributed control and estimation. We also showed that the effective resistances can be used to construct bounds on the spectrum of the Laplacian, Dirichlet Laplacian and other matrices that arise in distributed control and estimation. We examined a few novel and existing algorithms for such problems and showed that their fundamental performance limits, including stability and speed of convergence, can be determined by examining the effective resistances in the underlying network. Our coverage of problems for which effective resistances are relevant was not exhaustive and we encourage the readers to look for other problems where this tool can be of use.

\section{ACKNOWLEDGMENT}

The authors would like to thank Prof. Richard Murray for his comments that led to a tightening of the eigenvalue range in section IV-C.

\section{REFERENCES}

[1] P. Barooah and J. P. Hespanha. Optimal estimation from relative measurements: Electrical analogy and error bounds. Tech. rep., Center for Control, Dyn.Systems and Comp., Univ. of California, Santa Barbara, 2006.

[2] P. G. Doyle and J. L. Snell. Random Walks and Electric Networks. Math. Assoc. of America, 1984.

[3] A. K. Chandra, P. Raghavan, W. L. Ruzzo, R. Smolensky, et al.. The electrical resistance of a graph captures its commute and cover times. In Proc. of the Twenty First 21st Annual ACM Symposium on Theory of Computing, pp. 574-586. 1989.

[4] R. Karp, J. Elson, D. Estrin and S. Shenker. Optimal and global time synchronization in sensornets. Tech. rep., Center for Embedded Networked Sensing, Univ. of California, Los Angeles, 2003.

[5] P. Barooah and J. P. Hespanha. Estimation from relative measurements: Error bounds from electrical analogy. In Proc. of the 2nd Int. Conf. on Intelligent Sensing and Information Processing. 2005.

[6] A. Giridhar and P. R. Kumar. Distributed time synchronization in wireless networks: Algorithms and analysis (I). In 45th IEEE Conference on Decison and Control. 2006.

[7] P. Barooah and J. P. Hespanha. Distributed estimation from relative measurements in sensor networks. In Proc. of the 3nd Int. Conf. on Intelligent Sensing and Information Processing. 2005.

[8] A. J. Fax and R. M. Murray. Information flow and cooperative control of vehicle formations. IEEE Trans. on Automat. Contr., vol. 49, no. 9: 1465-1476, 2004.

[9] S. K. Yadlapalli, S. Darbha and K. R. Rajagopal. Information flow and its relation to the stability of the motion of vehicles in a rigid formation. In Proc. of the 2005 Amer. Contr. Conf., pp. 1853-1858. 2005.

[10] F. R. K. Chung. Spectral Graph Theory. No. 92 in Regional Conference Series in Mathematics. American Mathematical Society, Providence, R.I., 1997.

[11] C. Godsil and G. Royle. Algebraic Graph Theory. Graduate Texts in Mathematics. Springer, 2001.

[12] R. A. Horn and C. R. Johnson. Matrix Analysis. Cambridge University Press., Cambridge, 1993.

[13] R. Olfati-Saber, A. J. Fax and R. M. Murray. Consensus and cooperation in networked multi-agent systems. Proc. of IEEE, 2006. Submitted for publication.

[14] J. M. Mendel. Lessons in Digital Estimation Theory. Prentice Hall, New Jersey, 1987.

[15] P. Barooah and J. P. Hespanha. Error amplication and disturbance propagation in vehicle strings with decentralized linear control. In Proc. of the 44th Conf. on Decision and Contr. 2005. 